\title{
Reliability of ultrasound measurement of automatic activity of the abdominal muscle in participants with and without chronic low back pain
}

\author{
Amir Massoud Arab ${ }^{1 *}$, Omid Rasouli ${ }^{2}$, Mohsen Amiri ${ }^{1}$ and Nahid Tahan ${ }^{3}$
}

\begin{abstract}
Background: Ultrasound (US) imaging has been considered as a non-invasive technique to measure thickness and estimate relative abdominal muscle activity. Although some studies have assessed the reliability of US imaging, no study has assessed the reliability of US measurement of automatic activity of abdominal muscles in positions with different levels of stability in participants with chronic low back pain (cLBP). The purpose of this study was to investigate within-day and between-days reliability of US thickness measurements of automatic activity of the abdominal muscles in asymptomatic participants and within-day reliability in those with CLBP.

Methods: A total of 20 participants (10 with CLBP, 10 healthy) participated in the study. The reliability of US thickness measurements at supine lying and sitting positions (sitting on a chair, sitting on a gym ball with both feet on the ground or lifting one foot off the floor) were assessed. We evaluated within-day reliability in all participants and between-days reliability in asymptomatic participants.

Results: We found high ICC scores (0.85-0.95) and also small SEM and MDC scores in both groups. The reliability of the measurements was comparable between participants with and without LBP in each position but the SEMs and MDCs was slightly higher in patient group compared with healthy group. It indicates high intra-tester reliability for the US measurement of the thickness of abdominal muscles in all positions.
\end{abstract}

Conclusion: US imaging can be used as a reliable method for assessment of automatic activity of abdominal muscles in positions with low levels of stability in participants with and without LBP.

Keywords: Ultrasound, Reliability, Abdominal muscles, Low back pain

\section{Background}

Low back pain (LBP) is one of the most common musculoskeletal complaints in today's societies, affecting up to $70-80 \%$ of the population, at least one episode during their lifetime [1]. There is a considerable evidence indicating that dysfunction of abdominal muscles is a key impairment in patients with chronic LBP (cLBP) [2-4], which might affect spinal stability [5].

The abdominal musculature are divided into the anterolateral abdominal wall, consisting of the transversus abdominis (TrA), internal oblique (IO) and external oblique (EO); and the anterior wall, including the rectus

\footnotetext{
* Correspondence: arabloo_masoud@hotmail.com

'Department of Physical Therapy, University of Social Welfare and Rehabilitation Sciences, Evin, Koodakyar Ave, Zip Code: 1985713831 Tehran, Iran

Full list of author information is available at the end of the article
}

abdominis (RA) muscle and associated fascia. The TrA muscle and posterior part of IO muscle are the part of a deep muscle cylinder that play a major role in spinal stability during functional activities [6]. The RA and EO are considered as part of a global muscle system that control spinal orientation, balance the external loads applied to the trunk and transfer load from the thorax to the pelvis [7]. Automatic activation of the abdominal muscles has been considered as a protective mechanism for lumbar spine [8]. Different patterns of abdominal muscle activation have been reported in patients with LBP compared to healthy individuals [9-11]. Ferreira et al. [9] found changes in automatic control of TrA during isometric low load tasks with the limbs suspended in people with LBP. Rasouli et al. [11] showed although the thickness change in TrA and IO increased as the stability of the position

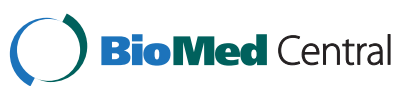


decreased in individuals with and without LBP, the amount of thickness changes were less in participants with LBP.

Assessment and measurement of the activity pattern of abdominal muscles can provide better understanding of pain behavior in patients with cLBP in the clinical environment.

A variety of measurement tools have been used by physical therapists to assess abdominal muscle activity in individuals with or without LBP. Real-time ultrasound (US) imaging has been recently used as a safe, costeffective and feasible method to evaluate muscle structure, function and activity [12-14]. It allows real time and direct visualization and evaluation of the abdominal muscles while they contract. Changes in the thickness of abdominal muscles is measured and considered as indicator of voluntary or automatic activity of the abdominal muscles during US imaging $[9,12,15]$. Validity studies found good to high correlation between the muscle thickness measured by US and EMG activity in low force contractions $[13,16]$ or those obtained using magnetic resonance imaging (MRI) [17]. McMeeken et al. [13] found good to high correlation between the needle EMG recordings of the TrA and US changes in thickness of the muscle at all activity level. Hodges et al. [16] compared the thickness changes in the abdominal muscles in real time US and EMG activity. They found that change in thickness and EMG activity of the TrA and IO muscles were linearly correlated at low contraction levels. Accordingly, using US imaging has been considered as a non-invasive technique to measure the thickness and estimate relative abdominal muscles activity.

Reliability is a psychometric property reflecting the degree to which repeated measurements provide similar results and reduction in measurement errors. Demonstrating acceptable reliability is essential for any kind of measurement and for making valid decisions. Considering lower stability, gym ball is frequently recommended in clinical practice to facilitate abdominal muscles activity. Change in the abdominal muscle thickness during sitting on a gym ball has been previously considered as automatic activity of the abdominal muscles to provide more stability $[11,18]$. Previous studies have investigated the reliability of US imaging in healthy individuals and patients with LBP [9,18-24] but they have mostly assessed the reliability of the antero-lateral abdominal wall muscles thickness, at rest and during voluntary abdominal muscle contraction such as abdominal hollowing manoeuvre [21,23,25-27]. The studies reported good to excellent reliability for single measures of thickness and poor to good reliability for thickness change measurements (reflecting the muscle activity). However, only a few studies evaluated the reliability of US measurements of automatic activation of abdominal muscles
$[9,12]$. Review of the literature showed that there is no research conducted to determine the reliability of US measurement of automatic activity of abdominal muscles in sitting positions with different levels of stability. Considering the fact that gym ball is not a stable condition and during sitting on gym ball the participant's position is instantaneously changing compared with sitting on a stable level such as a chair, abdominal muscles thickness may change in different moments and recording the US thickness measurement may be criticized.

Therefore, the purpose of this study was to evaluate within-day and between-days reliability in obtaining US thickness measurements of all abdominal muscles at supine lying and during different sitting positions (sitting on a chair, sitting on a gym ball with both feet on the ground or lifting one foot off the floor) in participants with and without cLBP.

\section{Methods}

\section{Subjects}

A total of 20 male volunteers (10 with chronic LBP, 10 without LBP) participated in the study. The population in this study was a sample of convenience made up of participants who were between the ages of 20 and 40 years. Patients were included if they had a history of LBP for more than six weeks before the study or had recurrent LBP and had experienced at least three episodes of LBP, each lasting more than one week, during the year before the study [28]. Asymptomatic participants were evaluated and found to have no complaint of any pain or dysfunction in their low back, pelvis, thoracic and lower extremities. Participants were excluded if they had a history of neuromuscular, musculoskeletal and cardiopulmonary diseases. The study conformed to the Declaration of Helsinki and was approved by the Human Ethics Committee at the University of Social Welfare and Rehabilitation Sciences, Tehran, Iran. All participants signed a consent form before participation in the study.

\section{Ultrasound measurement of the abdominal muscle thickness}

A diagnostic US imaging unit set in B-mode (Ultrasonix-ES500, Canada) with a linear array transducer (7.5 MHz) was used to measure the abdominal muscles thickness in four different positions: 1) supine lying, 2) relaxed sitting comfortably on a chair $43 \mathrm{~cm}$ high supported against a back rest, with arms folded; hands gently resting on the opposite shoulders and the feet together on the floor, 3) sitting comfortably with a straight back on a $65 \mathrm{~cm}$ in diameter, gym ball with arms folded and resting on the opposite shoulders and both feet on the floor and 4) sitting on a gym ball lifting the left foot off the floor by approximately $10 \mathrm{~cm}[11,18]$. Each position was held enough for the examiner to freeze a clear 
image of the muscle thickness at the end of expiration, usually no more than 2 minutes.

For antero-lateral abdominal wall muscles (TrA, IO, EO), the US transducer was transversely located across the right side of the abdominal wall over the anterior axillary line, midway between the 12th rib and the iliac crest, to obtain a clear image of the deep abdominal layers [13,22]. For anterior abdominal wall muscle (RA), the transducer was placed $2-3 \mathrm{~cm}$ above the umbilicus, $2-3 \mathrm{~cm}$ from the midline [22,29].

The image was frozen at the end of expiration and a vertical straight line through the center of the US image was used to ensure standardized placement of the measurement line. The cursor points carefully measured the muscle thickness between the inside edge of fascial bands in millimeter $(\mathrm{mm})$. The US transducer was not displaced during the testing procedure.

\section{Procedures}

Within-day intra-tester reliability of the US thickness measurements of the abdominal muscles was assessed in all participants (with and without cLBP) while betweenday reliability was investigated in asymptomatic participants. To establish within-day reliability, at first the examiner performed measurements in all tested positions in participants and then after 60 minutes repeated the measurements in a blinded fashion and in a random order with the same procedure. The participants testing positions and the order of measurements were randomly selected to reduce the memory effect. For between-days reliability, the US measurements in all positions were repeated after one week in asymptomatic participants $(\mathrm{N}=10)$. All testing procedure was performed in the biomechanics laboratory of the Department of Physical Therapy in the University of Social Welfare and Rehabilitation Sciences, Tehran, Iran.

\section{Data analysis}

Kolmogrov-Smirnov test was utilized to assess the normality of distribution for US measurement of muscle thickness at different testing positions. Normal distribution was observed for variables.

Two measurement sessions were implemented in one day for within-day reliability in all participants. Third session of measurement was done one week later in asymptomatic participants for between-days reliability assessment. The intra-class correlation coefficient (ICC) has become the preferred index for test-retest reliability as it reflects both correlation and agreement [30]. The ICC, two way mixed effect model, was used to assess intra-tester reliability of the measurements. We calculated the ICC $(3,1)$ as described by Shrout and Fleiss [31] because only one judge evaluated the same population.
Relative reliability values such as ICC are not sufficient to interpret in the context of an individual score. Therefore, we calculated standard error of measurement (SEM) and minimal detectable change (MDC) to make a judgment about the degree that measurements vary for an individual. The SEM is useful in reliability studies to determine the range of scores that would be expected from one assessment session to the next. The SEM was calculated using the formula: [SEM $=\mathrm{SD} \sqrt{ } 1-\mathrm{r}]$ where SD is the standard deviation calculated from the measurements and $r$ is the reliability coefficient for that measurement, in this case, the calculated ICC $(3,1)$ value and, then, the coefficient of variation was calculated (CV in \%: $\mathrm{SEM} /$ mean value for the given parameter $\times 100$ ) [21]

Power analysis was used to determine the sample size for test. Type I error $(\alpha)$ was set at 0.05 and power of the test was 0.80 . Considering this, it seems that calculated sample size in this study was appropriate to test the hypothesis and the results derived from the study are meaningful.

\section{Results}

The demographic data for both LBP and healthy control groups are summarized in Table 1.

Table 2 shows the US measurements of the absolute abdominal muscle thicknesses (Mean $\pm \mathrm{SD}$ ) in $\mathrm{mm}$ in two groups for different assessments during different testing positions. The smallest absolute abdominal thicknesses were related to supine lying position and the highest values belonged to position 4 when participant sat on the gym ball and lifted his left foot off the floor in both healthy and cLBP groups. The absolute thickness of TrA ranged from $3.5 \pm 0.5 \mathrm{~mm}$ to $6.6 \pm 0.8$ during four positions in healthy group and from $3.5 \pm 0.8$ to $4.9 \pm$ $1 \mathrm{~mm}$ in LBP group. The absolute thicknesses of IO,EO and RA ranged from $9.2 \pm 1.4$ to $12.8 \pm 2.1 ; 5.8 \pm 1.1$ to $7.5 \pm 0.9$ and $12.9 \pm 2$ to $16.9 \pm 2.2 \mathrm{~mm}$ during mentioned positions in healthy participants respectively, and from $8.3 \pm 1.1$ to $10.5 \pm 2.5 ; 5.8 \pm 1.1$ to $7.9 \pm 2.6$ and $14 \pm 3.7$ to $17.5 \pm 5.4 \mathrm{~mm}$ respectively in patients with LBP (Table 2).

Table 3 presents the ICC $(3,1)$, SEM, CV (SEM as \% mean) and MDC values for within-day and betweendays reliability of US thickness measurements of abdominal muscles were taken during different testing

Table 1 Demographic data of the participants in each group (Mean \pm SD)

\begin{tabular}{lcc}
\hline Variables & Without LBP $(\mathbf{n}=\mathbf{1 0})$ & With LBP $(\mathbf{n}=\mathbf{1 0})$ \\
\hline Age $($ years $)$ & $25 \pm 3$ & $26 \pm 3$ \\
Weight $(\mathrm{kg})$ & $75 \pm 7$ & $76 \pm 8$ \\
Height $(\mathrm{m})$ & $1.75 \pm 0.06$ & $1.75 \pm 0.06$ \\
BMI $\left(\mathrm{kg} / \mathrm{m}^{2}\right)$ & $24.3 \pm 0.9$ & $24.6 \pm 0.7$ \\
\hline
\end{tabular}

SD Standard deviation, LBP Low back pain, BMI Body mass index. 
Table 2 The (Mean \pm SD) scores for the ultrasound thickness measurements (in $\mathrm{mm}$ ) of the abdominal muscles during different testing positions in both groups

\begin{tabular}{|c|c|c|c|c|c|c|}
\hline \multirow[t]{2}{*}{ Position } & \multirow[t]{2}{*}{ Muscle } & \multicolumn{3}{|c|}{ Without LBP } & \multicolumn{2}{|c|}{ With LBP } \\
\hline & & First assessment & Second assessment & Third assessment & First assessment & Second assessment \\
\hline \multirow[t]{4}{*}{ P 1} & $\operatorname{TrA}$ & $3.6 \pm 0.6$ & $3.5 \pm 0.5$ & $3.8 \pm 0.6$ & $3.5 \pm 0.8$ & $3.7 \pm 1.0$ \\
\hline & 10 & $9.2 \pm 1.4$ & $9.3 \pm 1.3$ & $9.2 \pm 1.5$ & $8.4 \pm 1.5$ & $8.3 \pm 1.1$ \\
\hline & $\mathrm{EO}$ & $5.8 \pm 1.1$ & $5.9 \pm 1.2$ & $5.9 \pm 1.0$ & $5.8 \pm 1.1$ & $6.1 \pm 1.4$ \\
\hline & RA & $12.9 \pm 2.0$ & $13.0 \pm 2.3$ & $13.1 \pm 2.1$ & $14.0 \pm 3.7$ & $14.2 \pm 4.3$ \\
\hline \multirow[t]{4}{*}{ P 2} & $\operatorname{TrA}$ & $4.0 \pm 0.7$ & $3.9 \pm 0.8$ & $3.9 \pm 0.9$ & $3.6 \pm 0.8$ & $3.6 \pm 0.8$ \\
\hline & 10 & $9.4 \pm 1.5$ & $9.4 \pm 1.3$ & $9.5 \pm 1.8$ & $8.1 \pm 1.2$ & $8.3 \pm 1.3$ \\
\hline & EO & $6.2 \pm 1.2$ & $6.0 \pm 1.1$ & $5.9 \pm 1.2$ & $6.4 \pm 2.0$ & $6.7 \pm 2.0$ \\
\hline & RA & $13.1 \pm 2.0$ & $13.2 \pm 2.1$ & $13.4 \pm 2.0$ & $15.8 \pm 5.2$ & $15.3 \pm 4.8$ \\
\hline \multirow[t]{4}{*}{ P 3} & $\operatorname{TrA}$ & $4.4 \pm 0.7$ & $4.5 \pm 0.6$ & $4.4 \pm 0.5$ & $3.8 \pm 0.9$ & $4.0 \pm 0.7$ \\
\hline & 10 & $10.0 \pm 1.6$ & $10.0 \pm 1.7$ & $10.3 \pm 1.6$ & $8.8 \pm 2.2$ & $9.1 \pm 2.3$ \\
\hline & EO & $6.2 \pm 1.1$ & $6.54 \pm 1.3$ & $6.3 \pm 1.1$ & $6.6 \pm 2.2$ & $6.9 \pm 2.6$ \\
\hline & RA & $13.9 \pm 2.1$ & $14.1 \pm 2.4$ & $14.2 \pm 2.1$ & $16.2 \pm 5.4$ & $16.4 \pm 6.0$ \\
\hline \multirow[t]{4}{*}{ P 4} & $\operatorname{Tr} A$ & $6.6 \pm 0.8$ & $6.3 \pm 0.6$ & $6.5 \pm 0.8$ & $4.9 \pm 1.0$ & $4.1 \pm 1.0$ \\
\hline & 10 & $12.8 \pm 2.1$ & $12.8 \pm 2.1$ & $12.6 \pm 2.0$ & $10.5 \pm 2.5$ & $10.5 \pm 2.3$ \\
\hline & EO & $7.5 \pm 0.9$ & $7.4 \pm 1.2$ & $7.4 \pm 1.0$ & $7.9 \pm 2.6$ & $7.9 \pm 2.2$ \\
\hline & RA & $16.5 \pm 2.5$ & $16.8 \pm 2.8$ & $16.9 \pm 2.2$ & $17.2 \pm 4.8$ & $17.5 \pm 5.4$ \\
\hline
\end{tabular}

SD Standard deviation, LBP Low back pain, TrA Transversus abdominis, $I O$ Internal oblique, EO External oblique, $R A$ Rectus abdominis, $P 1$ Supine lying, $P 2$ Sitting on chair with both feet on the ground, $P 3$ Sitting on gym ball with both feet on the ground, $P 4$ Sitting on gym ball lifting the left foot off the floor.

positions in each group. Depending on the muscle (TrA, IO, EO and RA) and participant's position (supine lying or various sitting positions), reliability of thickness measurements ranged from 0.88 to 0.95 for within-day comparisons and from 0.85 to 0.94 for between-days comparisons in healthy group and from 0.89 to 0.94 for within-day comparisons in LBP group which indicated high intra-tester reliability for the US measurement of thickness of abdominal muscles in all positions (Table 3).

Generally we had higher SEM in position 4 than position 1 in both groups and SEMs of patient group (0.25 to $1.27 \mathrm{~mm}$ ) were rather higher than corresponding SEMs of control group ( 0.19 to $0.71 \mathrm{~mm}$ ), and, when expressed as a percentage $(\mathrm{CV})$ of the corresponding mean thicknesses ranged from 1.6 to $9.7 \%$ (in healthy participants) and 1.7 to $17.3 \%$ (in LBP patients) (Table 3).

Likewise SEM trends in two groups, MDCs were higher in position 4 than supine lying in both groups and MDCs of patient group ranged from 0.69 to $3.50 \mathrm{~mm}$ and were slightly higher than corresponding MDCs of control group (0.52 to $1.96 \mathrm{~mm}$ ) (Table 3).

\section{Discussion}

Physical therapists have recently utilized US imaging to evaluate muscle function, structure and activity and change in the thickness of abdominal muscles is measured and considered as indicator of voluntary or automatic activity of the abdominal muscles in this method $[12,14,15]$. However, like any other measurements demonstrating adequate reliability is a prerequisite for using US measurement as a valid measure of abdominal muscles activity to make decision especially in clinical setting. According to Domholdt [32], the reliability is not a fixed property but it depends on the studied population and testing positions.

To our knowledge, this is the first study to collectively evaluated within-day and between-days reliability of US measurement of automatic activity of abdominal muscles in sitting positions with different stability levels in healthy participants and within-day reliability in patients with cLBP.

The results of this study suggest that there is not only high reliability of muscle thickness in asymptomatic participants but also in patients with cLBP and the results also are consistent with the available literature on the topic, finding high ICC scores and also very small SEM and MDC scores [21,23].

In the patient group, the ICCs were almost the same as the control group during each testing position and it is important for using US to evaluate abdominal muscle activity in patients with LBP in the clinical setting. The ICCs of thickness measurements ranged from 0.85 to 0.95 for within-day and between-days comparisons depending on the muscle and participant's position which indicated high intra-tester reliability for the US 
Table 3 ICC, SEM and MDC, CV values for within-day and between-days reliability of the ultrasound measurements at different testing positions

\begin{tabular}{|c|c|c|c|c|c|c|c|c|c|c|c|c|c|}
\hline \multirow[t]{4}{*}{ Position } & \multirow[t]{4}{*}{ Muscle } & \multicolumn{8}{|c|}{ Without LBP } & \multirow{2}{*}{\multicolumn{4}{|c|}{$\begin{array}{c}\text { With LBP } \\
\text { Within-day }\end{array}$}} \\
\hline & & \multicolumn{4}{|c|}{ Within-day } & \multicolumn{4}{|c|}{ Between-days } & & & & \\
\hline & & \multicolumn{4}{|c|}{ (Between-trial) } & \multirow[b]{2}{*}{ ICC } & \multirow[b]{2}{*}{ SEM } & \multirow[b]{2}{*}{ MDC } & \multirow[b]{2}{*}{$\mathrm{CV} \%$} & \multicolumn{4}{|c|}{ (Between-trial) } \\
\hline & & ICC & SEM & MDC & $\mathrm{CV} \%$ & & & & & ICC & SEM & MDC & CV\% \\
\hline \multirow[t]{4}{*}{ P 1} & $\operatorname{Tr} A$ & 0.92 & 0.19 & 0.52 & 5.4 & 0.90 & 0.21 & 0.58 & 5.8 & 0.91 & 0.25 & 0.69 & 6.9 \\
\hline & 10 & 0.92 & 0.20 & 0.55 & 2.1 & 0.92 & 0.20 & 0.55 & 2.1 & 0.92 & 0.26 & 0.71 & 3 \\
\hline & EO & 0.90 & 0.23 & 0.63 & 3.9 & 0.88 & 0.26 & 0.71 & 4.5 & 0.89 & 0.34 & 0.93 & 5.7 \\
\hline & RA & 0.88 & 0.28 & 0.77 & 2.1 & 0.85 & 0.31 & 0.85 & 2.4 & 0.93 & 0.25 & 0.69 & 1.7 \\
\hline \multirow[t]{4}{*}{ P 2} & $\operatorname{TrA}$ & 0.95 & 0.30 & 0.82 & 7.6 & 0.92 & 0.38 & 1.05 & 9.7 & 0.94 & 0.37 & 1.02 & 10.2 \\
\hline & 10 & 0.93 & 0.40 & 1.10 & 4.5 & 0.93 & 0.40 & 1.10 & 4.2 & 0.93 & 0.39 & 1.07 & 4.8 \\
\hline & EO & 0.94 & 0.38 & 1.05 & 6.1 & 0.91 & 0.47 & 1.29 & 9 & 0.94 & 0.53 & 1.46 & 8.1 \\
\hline & RA & 0.93 & 0.52 & 1.43 & 3.9 & 0.93 & 0.52 & 1.43 & 3.9 & 0.94 & 0.56 & 1.54 & 3.6 \\
\hline \multirow[t]{4}{*}{ P 3} & $\operatorname{Tr} A$ & 0.90 & 0.34 & 0.93 & 7.7 & 0.86 & 0.40 & 1.10 & 9 & 0.90 & 0.39 & 1.07 & 10 \\
\hline & 10 & 0.92 & 0.31 & 0.85 & 3.1 & 0.90 & 0.34 & 0.93 & 3.4 & 0.93 & 0.49 & 1.35 & 5.3 \\
\hline & EO & 0.92 & 0.29 & 0.80 & 4.6 & 0.89 & 0.33 & 0.91 & 5.3 & 0.92 & 0.56 & 1.54 & 8.3 \\
\hline & RA & 0.93 & 0.22 & 0.60 & 1.6 & 0.87 & 0.31 & 0.85 & 2.2 & 0.93 & 0.65 & 1.79 & 4 \\
\hline \multirow[t]{4}{*}{ P 4} & $\operatorname{TrA}$ & 0.94 & 0.40 & 1.10 & 6.3 & 0.89 & 0.54 & 1.49 & 8.3 & 0.93 & 0.78 & 2.15 & 17.3 \\
\hline & 10 & 0.94 & 0.41 & 1.13 & 3.2 & 0.94 & 0.41 & 1.13 & 3.2 & 0.92 & 1.27 & 3.50 & 12 \\
\hline & EO & 0.92 & 0.52 & 1.43 & 7 & 0.93 & 0.48 & 1.32 & 6.4 & 0.92 & 1.23 & 3.39 & 15 \\
\hline & RA & 0.94 & 0.55 & 1.51 & 3.3 & 0.90 & 0.71 & 1.96 & 4.3 & 0.94 & 0.93 & 2.57 & 5.3 \\
\hline
\end{tabular}

SD Standard deviation, ICC Intra-class correlation coefficient, SEM Standard error of measurement, MDC Minimal detectable change, CV Coefficient of variation, LBP Low back pain, TrA Transversus abdominis, $I O$ Internal oblique, EO External oblique, RA Rectus abdominis, $P 1$ Supine lying, $P 2$ Sitting on chair with both feet on the ground, $P 3$ Sitting on gym ball with both feet on the ground, $P 4$ Sitting on gym ball lifting the left foot off the floor. All SEM and MDC values are in millimeter.

measurement of abdominal muscle thickness in all positions. Norasteh et al. [22] reported good reliability (ICC: 0.81 - 0.97) of abdominal muscles (TrA, IO, EO, RA) assessment by US imaging in asymptomatic participants and patients with acute LBP. In another study, Costa et al. [12] reported high reliability (ICC: 0.75- 0.94) for absolute thickness and moderate reliability (ICC: $0.26-$ 0.72 ) for thickness changes in US measurements of automatic activity of TrA, IO and EO muscles in patients with cLBP. However, in this study we assessed the reliability of absolute muscles thickness measurements and thickness changes were not analyzed.

In practical terms, the SEM shows more useful information than the ICC [33] and the CV is very good to compare the relative measurement error of variables with differing absolute values or measurement units. In the current study, the SEMs in position 4 were higher than position 1 in both groups and the SEMs of patient group $(0.25-1.27 \mathrm{~mm})$ were rather higher than corresponding SEMs of control group (0.19- $0.71 \mathrm{~mm})$, and, the CVs of the corresponding mean thicknesses ranged from 1.6 to $9.7 \%$ (healthy participants) and from 1.7 to $17.3 \%$ (cLBP patients). The SEMs were consistent with prior error measurements from other accepted techniques like Teyhen et al. [23] found the SEM of 0.13 to
$0.31 \mathrm{~mm}$ for the TrA muscle. The CV in this study was similar to the results of previous studies which reported $11-14 \%$ for the reliability of TrA thickness and whole abdominal mass thickness [21,23]. All the SEMs and the corresponding CVs were low, so make them promising measures for further studies.

The MDC is especially useful for interpreting the relevance of any changes recorded after an intervention [34]. The MDCs of abdominal muscles were higher in position 4 than supine lying in both groups, the same as SEM values and MDCs of patient group ranged from 0.7 to $3.5 \mathrm{~mm}$ and were slightly higher than corresponding MDCs of 0.5 to $1.9 \mathrm{~mm}$ in control group. Critchley et al. [35] reported MDC of $0.6(\mathrm{TrA})$ and $1.2(\mathrm{OI}) \mathrm{mm}$ in supine position, and Bunce et al. [36] found values ranging from 0.9 to $1.8 \mathrm{~mm}$ for TA only in supine, sitting and standing. The differences between our results with previous studies are related to the positions and level of contractions for example if we just exclude the values of position 4 , we have a range of 0.5 to $1.8 \mathrm{~mm}$. which are similar to those recorded at rest and hence appear to be acceptable from the perspective of human performance measurements [37].

Reliability can be influenced by several factors such as the participants, examiner and clinical or experimental 
setting. In addition, there are some sources of error which might affect procedure of US measurement including accuracy of marking the fascial bands, position of patient/transducer and detection of landmarks [12].

The high reliability of our US measurements may be due to the fact that the clear image was frozen on the screen and the muscle thickness between the fascial bands was measured carefully by cursor points. Furthermore, the US transducer was not displaced during the testing procedure as much as possible and the positions were the same for every participant.

This study was not investigated the issue of validity for US imaging. Considering the results of this study and validity studies $[16,17]$, US imaging can be used to assess and compare the automatic activity of the abdominal muscles in positions with different stability level between participants with and without cLBP.

\section{Conclusion}

In agreement with previous studies, the results of this study demonstrate high within-day and between-days reliability for US thickness measurements of the abdominal muscles at supine lying and sitting position with different levels of stability in healthy participants and high within-day reliability in patients with cLBP.

These results indicate that real-time US imaging can be reliably used to evaluate and compare the automatic activity of the abdominal muscles between participants with and without cLBP. This might also help to determine the efficacy of prescribed treatments for cLBP patients.

\section{Limitation}

This study assessed the reliability of US thickness measurements of the abdominal muscles in men. The results might not be extrapolated to the women. More studies are needed to collectively perform this study in men and women. The other point was that the study was only limited to a younger age group.

Another area of concern is that the between-day reliability was assessed in the asymptomatic participants. For between-days reliability, the US measurements were repeated after one week. It was possible that the pain intensity changed after one week in LBP patients. Change in pain intensity could affect the trunk stabilizer muscles such as abdominal muscles. Since the change in pain could not be completely controlled after one week, we assessed between-day reliability in asymptomatic participants with no LBP. This issue can be considered as a limitation in this study.

It is suggested to assess the within-day and betweenday reliability for automatic activation of the abdominal muscles during different tasks in the patients with cLBP.

\section{Clinical implication}

US imaging can be used as a reliable method to evaluate and compare the automatic activity of abdominal muscles in different positions with different levels of stability in participants with and without CLBP and this might help to determine the efficacy of prescribed treatments for cLBP patients.

\section{Ethical approval}

This research was reviewed and was approved by the Human Subject Committee at University of Social Welfare and Rehabilitation Sciences.

\section{Competing interests}

AMA contributed to conception, design, analysis, interpretation of data and drafting the manuscript. OR carried out the data collection and drafting the manuscript. MA participated in design and interpretation of data. NT participated in data collection and helped to draft the manuscript. All authors read and approved the final manuscript.

\section{Authors' contributions}

The authors declare that they have no competing interests.

\section{Author details}

${ }^{1}$ Department of Physical Therapy, University of Social Welfare and Rehabilitation Sciences, Evin, Koodakyar Ave, Zip Code: 1985713831 Tehran, Iran. ${ }^{2}$ Department of Physical Therapy, Faculty of Health Education and Social Work, Sør-Trøndelag University College (HiST), Trondheim, Norway. ${ }^{3}$ Department of Physical Therapy, Shahid Beheshti University of Medical Sciences, Tehran, Iran.

Received: 2 April 2013 Accepted: 29 October 2013

Published: 1 November 2013

\section{References}

1. Ehrlich GE: Low back pain. Bull World Health Organ 2003, 81:671-672.

2. Hodges PW, Richardson CA: Inefficient muscular stabilization of the lumbar spine associated with low back pain. A motor control evaluation of transverses abdominis. Spine 1996, 21:2640-2650.

3. Hodges PW, Richardson CA: Delayed postural contraction of transverses abdominis associated with movement of lower limb in people with low back pain. J Spinal Disord 1998, 11:46-56.

4. Jull GA, Richardson CA: Motor control problems in patients with spinal pain: a new direction for therapeutic exercises. J Manipulative Physiol Ther 2000, 23:115-117.

5. Cholewicki J, McGill SM: Mechanical stability of the in vivo lumbar spine: implications for injury and chronic low back pain. Clin Biomech 1996, 11:1-15.

6. Richardson CA, Jull GA, Hodges PW, Hides JA: Therapeutic exercise for spinal segmental stabilization in low back pain. Scientific basis and clinical approach. Edinburgh: Churchill Livingstone; 1999:43-70.

7. Bergmark A: Stability of the lumbar spine: a study in mechanical engineering. Acta Orthop Scand Suppl 1989, 230:1-54.

8. Hodges PW, Moseley GL: Pain and motor control of the lumbopelvic region: effect and possible mechanisms. J Electromyogr Kinesio/ 2003, 13:361-370.

9. Ferreira PH, Ferreira ML, Hodges PW: Changes in recruitment of the abdominal muscles in people with low back pain: Ultrasound measurement of muscle activity. Spine 2004, 29:2560-2566.

10. Hides J, Wilson S, Stanton W, McMahon S, Keto H, McMahon K, Bryant M, Richardson C: An MRI investigation into the function of the transversus abdominis muscle during "drawing-in" of the abdominal wall. Spine 2006, 31:E175-E178.

11. Rasouli O, Arab AM, Amiri M, Jaberzadeh S: Ultrasound measurement of deep abdominal muscle activity in sitting positions with different stability levels in subjects with and without chronic low back pain. Man Ther 2011, 16:388-393. 
12. Costa LO, Maher CG, Latimer J, Hodges PW, Shirley D: An investigation of the reproducibility of ultrasound measures of abdominal muscle activation in patients with chronic non-specific low back pain. Eur Spine $J$ 2009, 18:1059-1065.

13. McMeeken JM, Beith ID, Newham DJ, Milligan P, Critchley DJ: The relationship between EMG and changes in thickness of transverses abdominis. Clin Biomech 2004, 19:337-342.

14. Teyhen DS, Gill NW, Whittaker JL, Henry SM, Hides JA, Hodges P. Rehabilitative ultrasound imaging of the abdominal muscles. J Orthop Sports Phys Ther 2007, 37:450-466.

15. Hebert JJ, Koppenhaver SL, Parent EC, Fritz JM: A systematic review of the reliability of rehabilitative ultrasound imaging for the quantitative assessment of the abdominal and lumbar trunk muscles. Spine 2009, 34:E848-E856.

16. Hodges PW, Pengel LMH, Herbert RD, Gandevia SC: Measurement of muscle contraction with ultrasound imaging. Muscle and Nerve 2003, 27:682-692.

17. Hides JA, Belavý DL, Cassar L, Williams M, Wilson SJ, Richardson CA: Altered response of the anterolateral abdominal muscles to simulated weightbearing in subjects with low back pain. Eur Spine J 2009, 18:410-418.

18. Ainscough-potts AM, Morrissey MC, Critchley D: The response of the transverse abdiominis and internal oblique muscle to different postures. Man Ther 2006, 11:54-60.

19. Beazell JR, Grindstaff TL, Magrum EM, Cullaty M, Hart JM, Shen FH: Comparison of clinical test and real time ultrasound evaluation of muscle contraction in normals and patients with low back pain. J Man Manip Ther 2006, 14:168-169.

20. Koppenhaver SL, Hebert JJ, Fritz JM, Parent EC, Teyhen DS, Magel JS: Reliability of rehabilitative ultrasound imaging of the transversus abdominis and lumbar multifidus muscles. Arch Phys Med Rehabil 2009, 90:87-94.

21. Mannion AF, Pulkovski N, Gubler D, Gorelick M, O'Riordan D, Loupas T, Schenk P, Gerber H, Sprott H: Muscle thickness changes during abdominal hollowing: an assessment of between day measurement error in controls and patients with chronic low back pain. Eur Spine J 2008, 17:494-501.

22. Norasteh A, Ebrahimi E, Salavati M, Rafiei J, Abbasnejad E: Reliability of B-mode ultrasonography for abdominal muscles in asymptomatic and patients with acute low back pain. J Bodyw Mov Ther 2007, 11:17-20.

23. Teyhen DS, Miltenberger CE, Deiters HM, Del Toro YM, Pulliam JN, Childs JD, Boyles RE, Flynn TW: The use of ultrasound imaging of the abdominal drawing-in maneuver in subjects with low back pain. $J$ Orthop Sports Phys Ther 2005, 35:346-355.

24. Costa LO, Maher CG, Latimer J, Smeets RJ: Reproducibility of rehabilitative ultrasound imaging for the measurement of abdominal muscle activity: a systematic review. Phys Ther 2009, 89(8):756-769.

25. Pulkovski N, Mannion AF, Caporaso F, Toma V, Gubler D, Helbling D, Sprott $\mathrm{H}$ : Ultrasound assessment of transversus abdominis muscle contraction ratio during abdominal hollowing: a useful tool to distinguish between patients with chronic low back pain and healthy controls? Eur Spine J. Suppl 2012, 6:S750-S759.

26. Teyhen DS, Childs JD, Stokes MJ, Wright AC, Dugan JL, George SZ: Abdominal and lumbar multifidus muscle size and symmetry at rest and during contracted States. Normative reference ranges. J Ultrasound Med 2012, 31(7)1099-1110.

27. Teyhen DS, Rieger JL, Westrick RB, Miller AC, Molloy JM, Childs JD: Changes in deep abdominal muscle thickness during common trunkstrengthening exercises using ultrasound imaging. J Orthop Sports Phys Ther 2008, 38:596-605.

28. Nourbakhsh MR, Arab AM: Relationship between mechanical factors and incidence of low back pain. J Orthop Sports Phys Ther 2002, 32:447-460.

29. Misuri G, Colagrande S, Gorini M, landelli I, Mancini M, Duranti R, Scano G: In vivo ultrasound assessment of respiratory function of abdominal muscles in normal subjects. Eur Respir J 1997, 10:2861-2867.

30. Portney LG, Watkins MP: Reliability of measurements, Foundations of clinical research: applications to practice. 3rd edition. New Jersey: Pearson Education Inc; 2009.

31. Shrout PE, Fleiss JL: Intraclass correlation: use in assessing rater reliability. Psychol Bull 1979, 86:420-428.

32. Domholdt E: Rehabilitation research: principles and applications. 3rd edition. Philadelphia: Elsevier Saunders; 2005.
33. Rankin G, Stokes M: Reliability of assessment tools in rehabilitation: an illustration of appropriate statistical analyses. Clin Rehabil 1998, 12:187-199

34. Beaton DE: Understanding the relevance of measured change through studies of responsiveness. Spine 2000, 25:3192-3199.

35. Critchley DJ, Coutts FJ: Abdominal muscle function in chronic low back pain patients. Physiotherapy 2002, 88:322-332.

36. Bunce $\mathrm{SM}$, Moore $\mathrm{AP}$, Hough $\mathrm{AD}$ : M-mode ultrasound: a reliable measure of transversus abdominis thickness? Clin Biomech (Bristol, Avon) 2002, 17:315-317.

37. Stokes M: Reliability and repeatability of methods for measuring muscle in physiotherapy. Physiother Theory Pract 1985, 1:71-76.

doi:10.1186/2045-709X-21-37

Cite this article as: Arab et al:: Reliability of ultrasound measurement of automatic activity of the abdominal muscle in participants with and without chronic low back pain. Chiropractic \& Manual Therapies 2013 21:37.

\section{Submit your next manuscript to BioMed Central and take full advantage of:}

- Convenient online submission

- Thorough peer review

- No space constraints or color figure charges

- Immediate publication on acceptance

- Inclusion in PubMed, CAS, Scopus and Google Scholar

- Research which is freely available for redistribution

Submit your manuscript at www.biomedcentral.com/submit
C Biomed Central 